Revista Energia na Agricultura

ISSN 1808-8759

\title{
DESENVOLVIMENTO E AVALIAÇÃO DE DESEMPENHO DE UM SISTEMA DE POSICIO- NAMENTO AUTOMÁTICO PARA PAINÉIS FOTOVOLTAICOS ${ }^{1}$ \\ ALCEU FERREIRA ALVES ${ }^{2} \&$ JOSÉ ANGELO CAGNON ${ }^{3}$
}

RESUMO: A necessidade de utilização da energia elétrica em localidades não atendidas pelas redes convencionais de distribuição, com eficiência, confiabilidade e custo acessível, motivou a realização desta pesquisa, cujo enfoque principal foram os sistemas fotovoltaicos e a busca por um melhor desempenho destes sistemas a partir de estratégias de posicionamento dos painéis solares em direção ao Sol. Este trabalho apresenta o desenvolvimento de um conjunto posicionador para painéis fotovoltaicos e a avaliação do desempenho deste conjunto, considerando-se o aumento na geração de energia e seus custos de implantação. Projetou-se um dispositivo eletromecânico simplificado, capaz de sustentar e movimentar um painel fotovoltaico ao longo do dia e ao longo do ano, mantendo sua superfície direcionada em posição perpendicular aos raios solares, sem uso de sensores e com otimização dos movimentos, devido ao ajuste de inclinação do painel ser realizado somente uma vez ao dia. Os resultados obtidos permitiram concluir que a proposta é viável, demonstrando melhor aproveitamento da energia solar e custo compatível com o ganho obtido na geração de eletricidade.

Palavras-chave: Sistemas fotovoltaicos, energia solar, fontes alternativas de energia.

\footnotetext{
${ }^{1}$ Extraído da Tese de Doutorado do primeiro autor, intitulada: "Desenvolvimento de um Sistema de Posicionamento Automático para Painéis Fotovoltaicos”.

${ }^{2}$ Aluno do Programa de Pós-Graduação em Agronomia - Energia na Agricultura - FCA/UNESP, Botucatu/SP e Docente do Departamento de Engenharia Elétrica - FEB/UNESP, Bauru/SP, alceu@ feb.unesp.br

${ }^{3}$ Orientador, Docente do Programa de Pós-Graduação em Agronomia - Energia na Agricultura - FCA/UNESP, Botucatu/SP e Docente do Departamento de Engenharia Elétrica - FEB/UNESP, Bauru/SP, jacagnon@ feb.unesp.br
} 


\section{DEVELOPMENT AND PERFORMANCE EVALUATION OF AN AUTOMATIC POSITIONING SYSTEM FOR PHOTOVOLTAIC PANELS}

SUMMARY: The need of using electric energy in localities not attended by the utilities, with efficiency, reliability and accessible cost, has motivated the development of this research, which main approach was photovoltaic systems and the search for better performance of these systems with the solar panels positioning toward the sun. This work presents the experimental development of an automatic positioning system for photovoltaic panels and the evaluation of this system's behavior taking in account the increase in generation of electric energy and its costs of implantation. It was designed a simplified electromechanical device, which is able to support and to move a photovoltaic panel all day long and all year long, keeping its surface aimed to the sun rays, without using sensors and with optimization of movements, due to the adjustment of panel's inclination take place only once a day. The obtained results indicated that the proposal is viable, demonstrating better utilization of the solar energy, showing a compatible cost compared to the increase in the generation of electricity.

Keywords: Photovoltaic systems, solar energy, energy - renewable sources.

\section{INTRODUÇÃO}

A energia elétrica é uma das formas mais versáteis e convenientes de energia, sendo recurso indispensável e estratégico para o desenvolvimento das populações. Apesar dos avanços tecnológicos e benefícios proporcionados pela eletricidade, cerca de um terço da população mundial ainda não tem acesso a esse recurso, e uma parcela considerável dos dois terços restantes é atendida de forma muito precária. No Brasil, a situação é menos crítica, mas ainda preocupante. Embora exista abundância de recursos energéticos, há grande diversidade regional e uma forte concentração de pessoas e atividades econômicas em regiões com problemas de suprimento energético (ATLAS, 2005).

Para atingir populações isoladas ou em regiões de difícil acesso às redes de distribuição convencionais, a energia solar fotovoltaica apresenta-se como uma solução emergente. Segundo a Empresa de Pesquisa Energética, através do Plano Nacional de Energia 2030 (Brasil, 2007), a energia fotovoltaica é uma grande promessa para a geração distribuída. Um dos aspectos importantes ressaltados é que o seu 
emprego tem contribuído para normalizar questões essenciais da geração distribuída, nos aspectos da qualidade, segurança e proteção. Outro fator importante na expansão do uso da tecnologia fotovoltaica é o apelo ambiental, pois estes sistemas têm como características a não modificação do equilíbrio térmico da Terra, operando sem emissão de gases de efeito estufa ou de gases ácidos, não sendo necessária a formação de lagos para reservatórios, e o desmatamento só ocorre em grandes instalações, as quais, muitas vezes são construídas em áreas desérticas ou semi-áridas.

Pesquisas que buscam o aumento da eficiência nos sistemas fotovoltaicos têm sido desenvolvidas e enfocam diferentes alternativas para alcançar este objetivo. As variadas tecnologias de construção das células fotovoltaicas têm resultado em melhor eficiência na conversão da energia solar. Nas últimas três décadas, avanços significativos no desenvolvimento de materiais permitiram o aumento da eficiência dos painéis, com diminuição de custos, tornando a tecnologia acessível a uma maior parcela da população. Eficiências superiores a $20 \%$ têm sido obtidas com células de silício produzidas em larga escala.

O uso de posicionadores automáticos para os painéis solares é uma alternativa para oferecer maior quantidade de energia solar ao painel, permitindo maior geração de potência elétrica.

\subsection{Sistemas de Posicionamento}

Segundo Chun-Sheng et al. (2008, p. 2586), "nas atuais condições de desenvolvimento da tecnologia, a utilização de sistemas fotovoltaicos com rastreamento solar é uma ótima solução para o aumento na eficiência e redução nos custos”. Estes sistemas, conhecidos como Solar Tracking, procuram colocar os painéis solares com a superfície sempre em posição perpendicular aos raios de Sol incidentes. Desta maneira, as mudanças na posição do Sol em relação à Terra durante o dia e com respeito às estações do ano são compensadas com o reposicionamento do módulo, permitindo que mais energia seja coletada e transformada em eletricidade dentro de um determinado intervalo de tempo (MARINESCU; MARINESCU, 2006).

Ainda de acordo com Chun-Sheng et. al, as pesquisas demonstram que sistemas que rastreiam o movimento do Sol e trabalham em um único eixo (single axis) podem aumentar em mais de $20 \%$ a produção de eletricidade, enquanto que sistemas de rastreamento em dois eixos (double axis) podem aumentar em mais de $40 \%$ a produção de eletricidade.

Plantas de geração de energia elétrica com tecnologia fotovoltaica instaladas em desertos, conhecidas como "Sistemas Fotovoltaicos de Larga Escala (VLS-PV)" foram objetos de estudos e os resultados relatados comprovam sua viabilidade técnica e econômica. Ito et al. $(2003,2004)$ apresentam os custos ao longo do ciclo de vida para sistemas montados sobre estruturas fixas, estruturas móveis em 1 eixo e estru- 
turas móveis de rastreamento em 2 eixos. Em suas conclusões, demonstram a viabilidade econômica de um sistema fotovoltaico, com rastreamento, para uma usina geradora de 100MW.

Marinescu e Marinescu (2006) apresentaram uma avaliação bastante detalhada de sistemas de posicionamento utilizando as mais diversas tecnologias. Sistemas que utilizam sensores, em geral, necessitam de um dispositivo eletrônico que possa avaliar a melhor posição e comandar o movimento do(s) módulo(s). Estes sistemas apresentam em comum a limitação de funcionamento sob condições 'parasitas' ou 'não previstas', como por exemplo, a ocorrência parcial de nuvens no céu ou sombras que encubram parcialmente um ou mais sensores, causando erros no rastreamento.

Um estudo sobre diferentes tipos de algoritmos utilizados para sistemas de controle de rastreamento foi desenvolvido por Chojnacki e Teneta (2004). Neste, os autores utilizam um sistema mecânico padrão, cujo esquema cinemático é apresentado na Figura 1.

Comparando um sistema fixo e um sistema móvel com as mesmas características elétricas, foi observado um aumento de aproximadamente $22 \%$ na geração de energia elétrica utilizando-se um algoritmo com elevado grau de complexidade, que combina cálculos baseados no tempo com indicações do sensor direcional de radiação e um discriminador do nível de insolação. Os intervalos de tempo de reposicionamento do painel são definidos a partir do máximo erro de posicionamento permitido para o painel fotovoltaico (CHOJNACKI; TENETA, 2004).

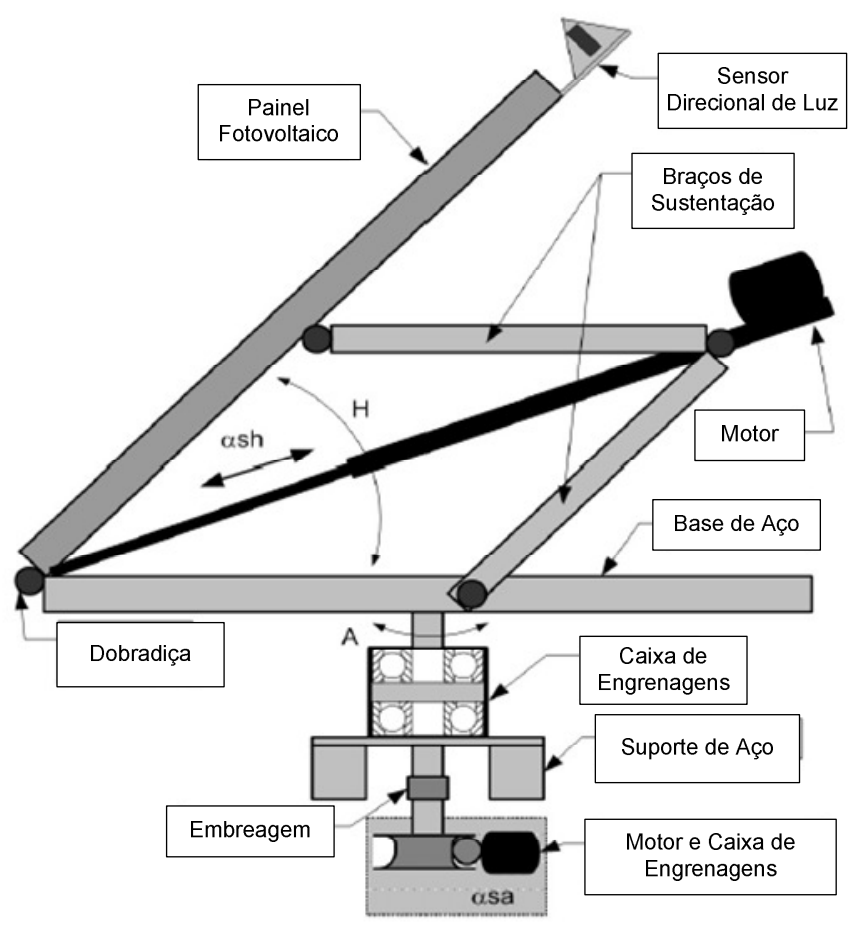

Figura 1 - Diagrama cinemático do Sistema de Rastreamento.

Fonte - Chojnacki e Teneta, 2004 


\section{MATERIAL E MÉTODOS}

Para avaliação da proposta comparou-se o funcionamento de um sistema fixo e um sistema móvel. Foi construído um Laboratório de Campo na cidade de Bauru/SP, com localização geográfica $22^{\circ} 21^{\prime}$ (S) e $49^{\circ} 01^{\prime}(\mathrm{W})$, com altitude de 613 metros acima do nível do mar. Constituiu-se de uma base plana, retangular, construída em concreto e cercada com alambrado, sobre a qual foram instalados dois armários de aço que abrigam os equipamentos; sobre estes armários foram instalados os dois painéis fotovoltaicos, um fixo e outro móvel. A Figura 2 apresenta fotos deste Laboratório.
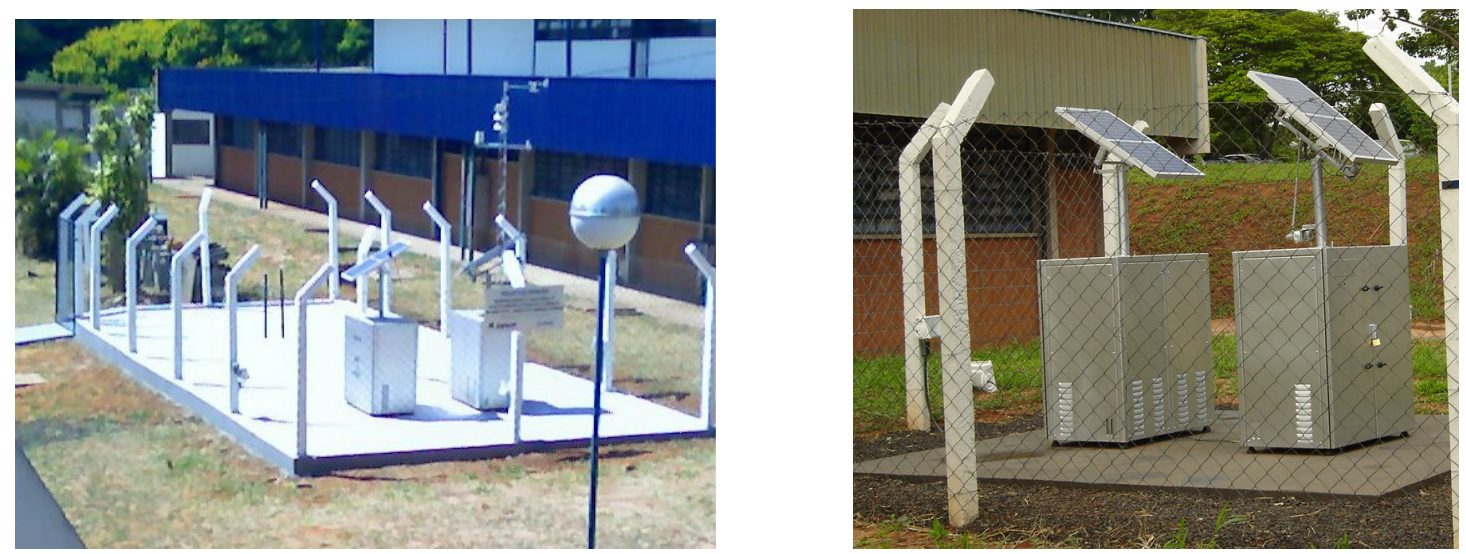

Figura 2 - Laboratório Experimental.

O diagrama de blocos da Figura 3 representa o sistema experimental completo. Neste diagrama podem ser vistos os painéis fotovoltaicos sobre os seus suportes (fixo e móvel), os sensores de radiação e temperatura, os motores, e as cargas resistivas. Ao centro do experimento está o Controlador Lógico Programável (CLP), que controla o sistema móvel e também coleta e armazena os dados dos sensores.

Os dois painéis fotovoltaicos são idênticos, de silício monocristalino, com as seguintes características nominais (tolerâncias de $+/-10 \%$ ):

$$
\begin{array}{ll}
\text { - } & \text { Tensão de Circuito Aberto }\left(V_{O C}\right)=21,3 \mathrm{~V} \\
\text { - } & \text { Corrente de Curto-Circuito }\left(I_{S C C}\right)=3,4 \mathrm{~A} \\
\text { - } & \text { Tensão de Potência Máxima }\left(V_{M P}\right)=16,7 \mathrm{~V} \\
\text { - } & \text { Corrente de Potência Máxima }\left(I_{M P}\right)=3,0 \mathrm{~A} \\
& \text { Potência Máxima }\left(P_{M}\right)=50 \mathrm{Wp}
\end{array}
$$


O painel fixo está montado sobre um suporte mecânico padrão, direcionado para o Norte Solar, permitindo do ajuste manual da inclinação ao longo do ano. Ao suporte do outro painel estão adaptados os motores que realizam ajustes automáticos de ângulo horário (Azimute) e inclinação (vide Figura 4).

Para avaliação da geração de energia, ambos os painéis alimentaram cargas resistivas de valor fixo, com potência superior à capacidade de geração do módulo fotovoltaico. As cargas utilizadas eram lâmpadas incandescentes de $12 \mathrm{~V} / 55 \mathrm{~W}$, idênticas para ambos os painéis.

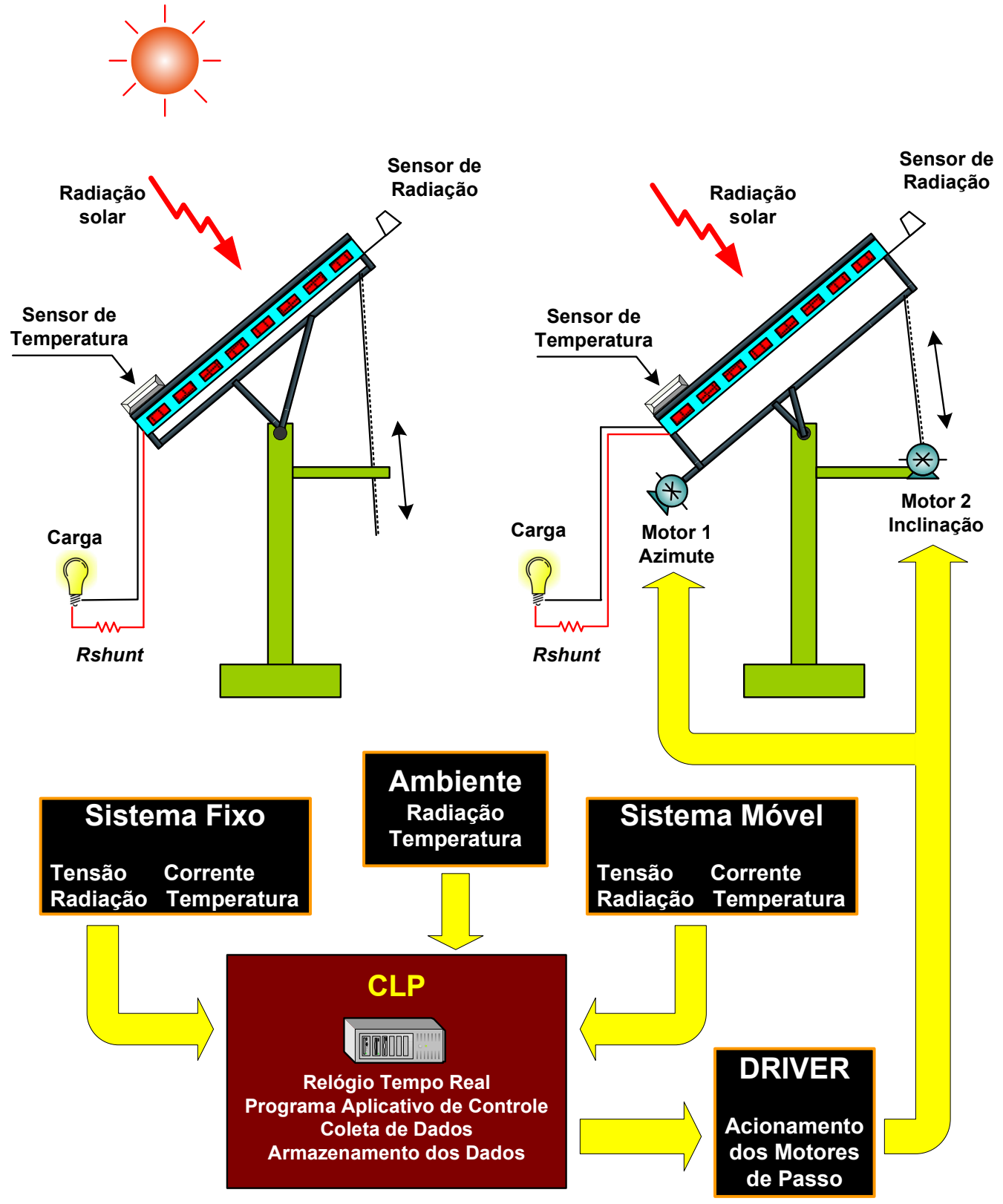

Figura 3 - Diagrama completo do sistema experimental desenvolvido. 


\subsection{Características do Sistema de Posicionamento}

O sistema de posicionamento era composto pelo Controlador Lógico Programável, placa de acionamento dos motores de passo, motores, e redutores. O CLP executava o programa de controle que calculava a posição aparente do Sol no céu e gerava os sinais de controle para os motores. Estes sinais de controle são transformados nos pulsos que acionam os motores, determinando a velocidade, a direção do movimento e os ângulos de posicionamento.

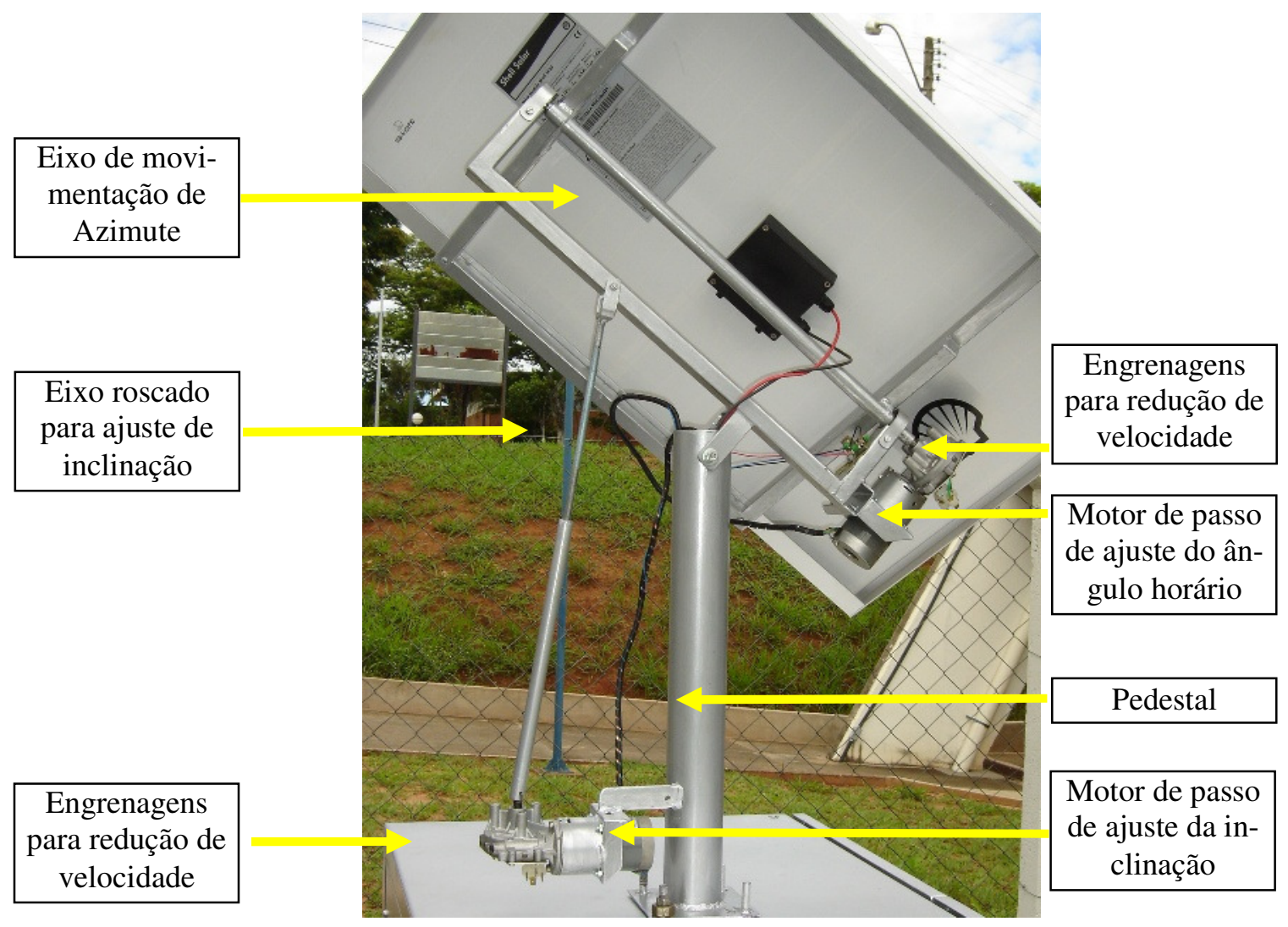

Figura 4 - Detalhe do suporte mecânico de sustentação do Painel Móvel.

Os dispositivos atuadores eram motores de passo de baixa potência acoplados a redutores mecânicos. Eram motores de passo com tensão nominal de $7 \mathrm{~V}$, corrente máxima de 0,34 A (eixo bloqueado), resistência de cada bobina de $21 \Omega$, torque máximo de $5 \mathrm{kgf}$ e precisão de $1,8^{\circ}$.

\subsection{Descrição dos Sensores}

Os parâmetros coletados foram: tensão e corrente em cada painel, temperatura e radiação solar. A 
tensão foi medida diretamente nos terminais dos painéis fotovoltaicos em intervalos de 1 minuto. A medida da corrente foi feita de maneira indireta, amostrando-se a tensão sobre um resistor conectado em série com cada uma das cargas (shunt), também em intervalos de 1 minuto.

O resistor shunt escolhido foi composto de um suporte de latão sobre o qual são apoiados dois fios de manganina (ou manganin), uma liga composta por $86 \%$ de Cobre, $12 \%$ de Manganês e $2 \%$ de Níquel. Possuia estabilidade térmica e boa linearidade, na faixa de 0 a $10 \mathrm{~A}$. Seu valor nominal foi $0,015 \Omega$, resultando em $150 \mathrm{mV}$ para corrente nominal de 10A.

A medida de temperatura foi feita por sensores resistivos instalados na superfície de ambos os painéis e também dentro do armário de aço. O sensor utilizado foi o PT-100, sensor de platina a 3 fios para medida de temperaturas na faixa de $-200^{\circ} \mathrm{C}$ a $650^{\circ} \mathrm{C}$, e fornecia sinal de resistência diretamente aplicável a uma entrada do CLP específica para esta finalidade (

Figura 5).
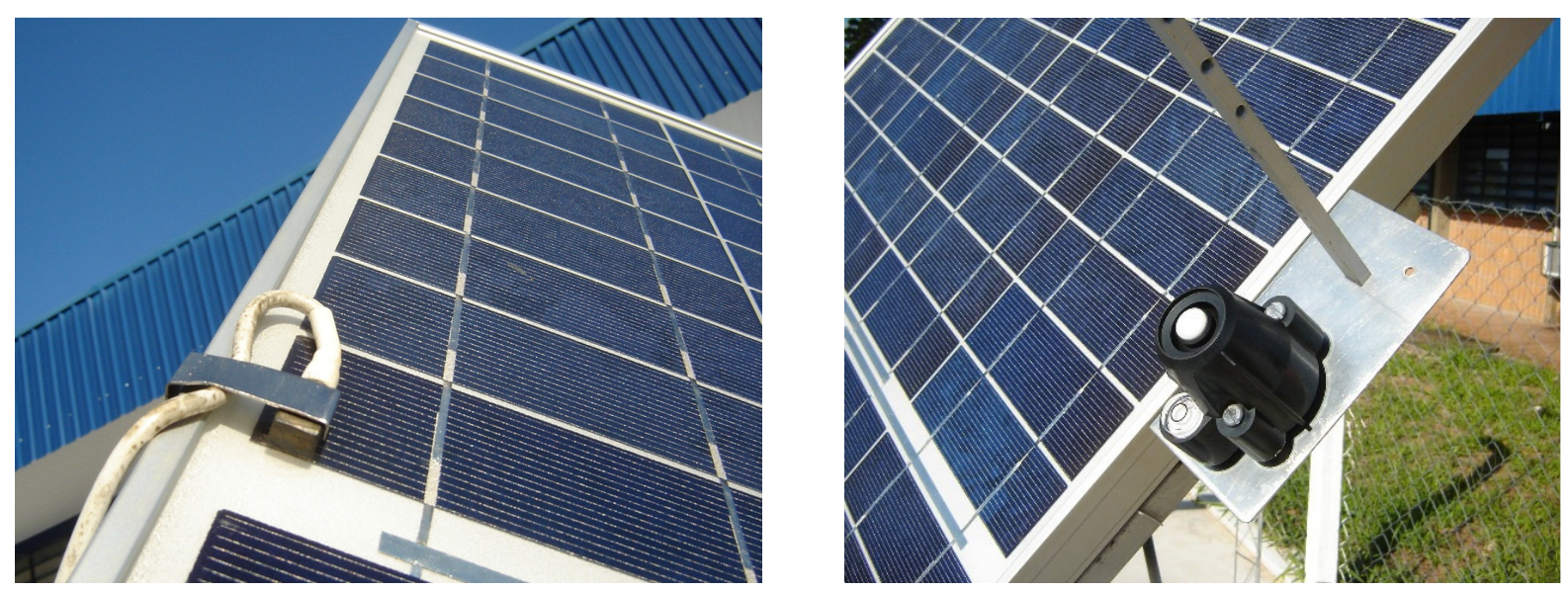

(a) Sensor de Temperatura

(b) Sensor de Radiação Global

Figura 5 - Sensores de Temperatura e Radiação.

Os valores de radiação foram obtidos a partir de dois sensores colocados nos planos dos painéis (vide

Figura 5) e um terceiro sensor em posição horizontal em relação ao solo, para medida da radiação solar global incidente no ambiente. O sensor escolhido foi um piranômetro do fabricante Davis Instruments, capaz de detectar radiação de comprimentos de onda entre $0,3 \cdot \mathrm{m}$ a $1100 \cdot \mathrm{m}$, que utilizava como detector um fotodiodo de silício. 


\subsection{Placa de Acionamento dos Motores de Passo}

Projetou-se e construiu-se uma placa que recebia os comandos do CLP e os transformava em pulsos para acionamento dos motores. Esta placa utilizava o controlador L297 (Stepper Motor Controller) e a “ponte H” L298N (Dual Full Bridge Driver), além de um conjunto de 8 diodos para proteção das saídas do circuito integrado contra pulsos de tensão excessivamente elevados.

\subsection{Desenvolvimento do Sistema de Controle e Monitoramento}

O programa de controle e movimentação do painel era constituído de uma rotina principal, a qual 'chama' as sub-rotinas de cálculos, de leitura dos sensores, de acionamento dos motores e de armazenamento de dados. As rotinas de leitura e conversão dos parâmetros de entrada que foram utilizados pelas rotinas de cálculos estão ilustradas na Figura 6.

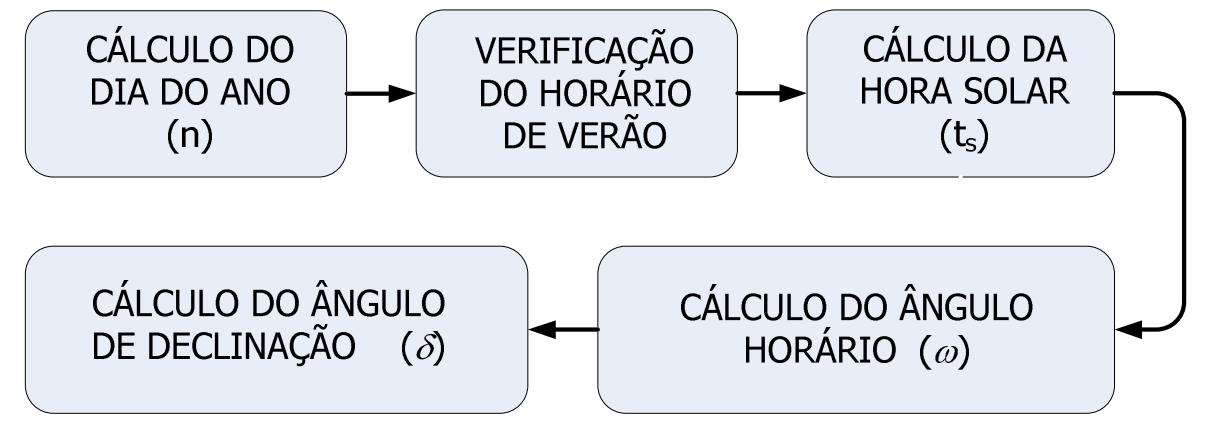

Figura 6 - Diagrama em Blocos das Rotinas de Cálculos.

O primeiro passo foi encontrar a relação entre a hora solar $\left(t_{s}\right)$ e a hora dos relógios locais (LCT $=$ Local Clock Time). Para isto, calculou-se o dia do ano (n) convertendo-se o dia do ano Juliano em um somatório unitário, desde o primeiro até o último dia do ano em um ciclo de 4 anos. Este resultado foi utilizado para o cálculo da Equação do Tempo (EOT) e de LC (Correção da Longitude Local), parâmetros necessários para o cálculo dos ângulos de azimute (•) e de declinação ( • • ). As equações (1), (2), (3),

(4) e (5) resumem os cálculos realizados pelo CLP e necessários para as rotinas de movimentação do painel.

$$
E O T=60 \sum_{k=0}^{5}\left[A_{k} \cos \left(\frac{360 k n}{365,25}\right)+B_{k} \operatorname{sen}\left(\frac{360 k n}{365,25}\right)\right]
$$




$$
L C=\frac{(\text { longitude local })-\left(\begin{array}{c}
\text { longitude do meridiano } \\
\text { do fuso horário local }
\end{array}\right)}{15}
$$

(horas)

(2)

$$
t_{S}=L C T+\frac{E O T}{60}-L C-D
$$

$$
\omega=15\left(t_{s}-12\right)
$$

(graus)

$$
\operatorname{sen} \delta=0,39795 \cos [0,98563(n-173)]
$$

Para o cálculo da Equação do Tempo são necessários os Coeficientes de Stine e Geyer, apresentados na Tabela 1. O cálculo da hora solar (Equação 3) necessita do parâmetro $D$, o qual é inserido via teclado para informar se a hora dos relógios locais está ou não no período de horário de verão.

Tabela 1 - Coeficientes da Equação (1).

\begin{tabular}{ccc}
\hline $\boldsymbol{K}$ & $\boldsymbol{A}_{\boldsymbol{k}}($ hora $)$ & $\boldsymbol{B}_{\boldsymbol{k}}$ (hora) \\
\hline 0 & $2,0870 \times 10^{-4}$ & 0 \\
1 & $9,2869 \times 10^{-3}$ & $-1,2229 \times 10^{-1}$ \\
2 & $-5,2258 \times 10^{-2}$ & $-1,5698 \times 10^{-1}$ \\
3 & $-1,3077 \times 10^{-3}$ & $-5,1602 \times 10^{-3}$ \\
4 & $-2,1867 \times 10^{-3}$ & $-2,9823 \times 10^{-3}$ \\
5 & $-1,5100 \times 10^{-4}$ & $-2,3463 \times 10^{-4}$ \\
\hline
\end{tabular}

Fonte: Stine e Geyer, 2001 
As rotinas de movimentação do painel estão descritas pelo Diagrama em Blocos da

Figura 7. Antes do amanhecer, o painel está posicionado ao centro de sua trajetória; a rotina de Movimento Inicial 1 desloca o painel desta posição até o sensor de início de curso. Trata-se de uma rotina de calibração, uma vez que o sensor de início de curso tem posição fixa e conhecida em relação ao plano horizontal.

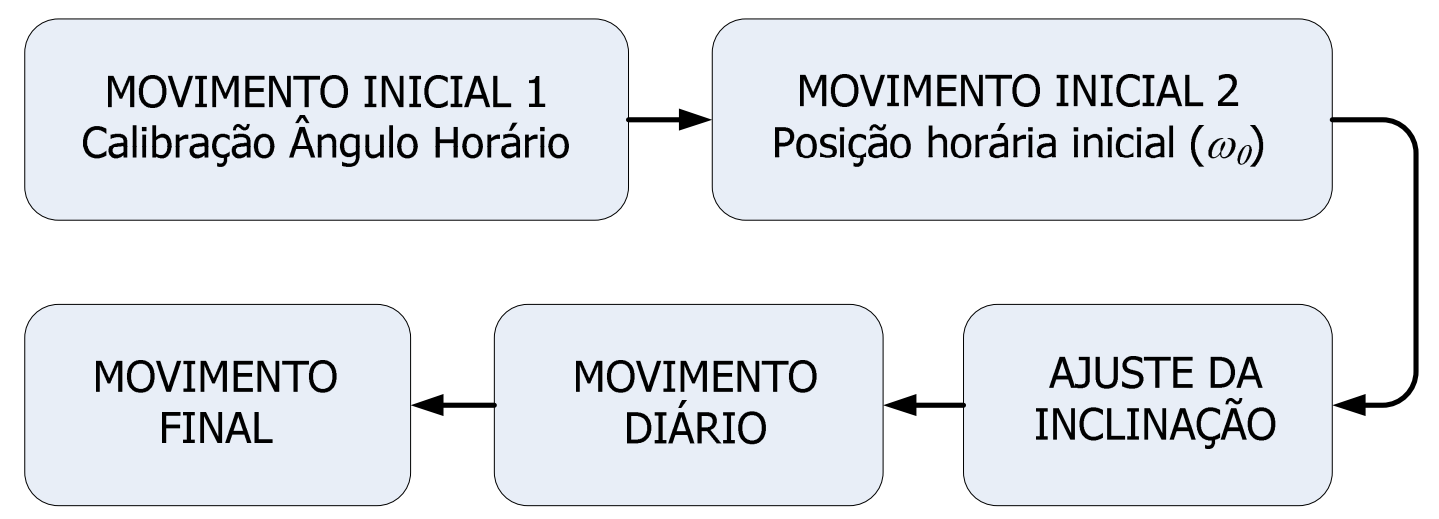

Figura 7 - Diagrama em Blocos das Rotinas de Movimentação.

Em seguida, a rotina de Movimento Inicial 2 gera os pulsos necessários para que o motor de movimentação de Azimute leve o painel até a posição inicial de movimento.

A rotina de movimento anual atua no motor de movimentação do eixo de inclinação do painel, e a partir do valor calculado para o ângulo de declinação, corrige a inclinação caso seja necessário.

Sabendo-se que o Sol, em seu movimento aparente, desloca-se $15^{\circ}$ em uma hora, determinou-se o movimento do painel de $1^{\circ}$ a cada 4 minutos. A rotina de movimento diário se encarregava de fazer este movimento, começando no horário programado, no qual o painel estava posicionado perpendicularmente aos raios de Sol incidentes. Com o deslocamento de $1^{\circ}$ a cada 4 minutos, há um sincronismo entre o movimento do painel e o movimento aparente do Sol, o que mantém a superfície do painel em posição perpendicular aos raios de Sol incidentes durante todo o período, até que o painel atinja o sensor de fim de curso, cessando assim sua movimentação diária.

Após o pôr-do-sol, em um horário pré-determinado, realizava a movimentação de retorno do painel até este ficar posicionado ao centro de sua trajetória, onde aguardaria o início do próximo dia. 


\subsection{Coleta dos Dados Experimentais}

A rotina de coleta de dados implementada no CLP registrava as leituras dos dados em intervalos de 1 minuto, no período compreendido entre $7 \mathrm{~h} 00$ e $17 \mathrm{~h} 00$ ( $L C T$ ), resultando em tabelas diárias de 600 linhas (10 horas, 60 leituras por hora) e 11 colunas, contendo: Hora do dia (tempo), Tensão do Painel Móvel (VM) e do Painel Fixo (VF), Corrente do Painel Móvel (IM) e do Painel Fixo (IF), Temperatura Ambiente (TA), da Superfície do Painel Móvel (TM) e da Superfície do Painel Fixo (TF), Irradiância no Plano do Painel Móvel (RM), no Plano do Painel Fixo (RF) e no Plano Horizontal (RA). Ao final do dia, esta tabela de 6.600 pontos foi transferida a um computador pessoal.

\section{RESULTADOS E DISCUSSÃO}

Os dados experimentais foram coletados durante um período de 152 dias, que compreendeu épocas de tempo seco, épocas de tempo chuvoso, dias frios e dias mais quentes, período de verão, outono e inverno, e permitiu uma avaliação do ganho de rendimento em condições bastante variadas de operação do sistema fotovoltaico móvel em comparação com o sistema fixo.

A Figura 8 apresenta as curvas de potência entregue à carga (equivalente à potência instantânea gerada, considerando perdas desprezíveis entre a geração e o consumo) para os dois painéis fotovoltaicos, trabalhando em condições idênticas de operação, no dia 26/06/2008.

Nas curvas traçadas verifica-se o comportamento esperado para o painel fixo: a potência gerada no início do dia é zero antes do nascer do Sol, e aumenta gradativamente a partir das 7h36min, atingindo o seu máximo por volta das $12 \mathrm{~h} 37 \mathrm{~min}$, próximo ao meio-dia solar. Observa-se que durante o período da manhã o céu esteve claro, com poucas interferências de nuvens ou sombras, o que se alterou ligeiramente no período da tarde, quando se observam variações um pouco mais acentuadas na curva de potência.

O comportamento do painel fotovoltaico móvel evidencia seu melhor desempenho quando se trata da potência instantânea. Desde o início do dia, a potência gerada por este painel é substancialmente maior que a potência do painel fixo. Às $9 \mathrm{~h} 31 \mathrm{~min}$ esta diferença atinge $150 \%$, ou seja, a potência instantânea do painel móvel é 26,93 W enquanto que a potência instantânea do painel fixo é 10,73 W. 


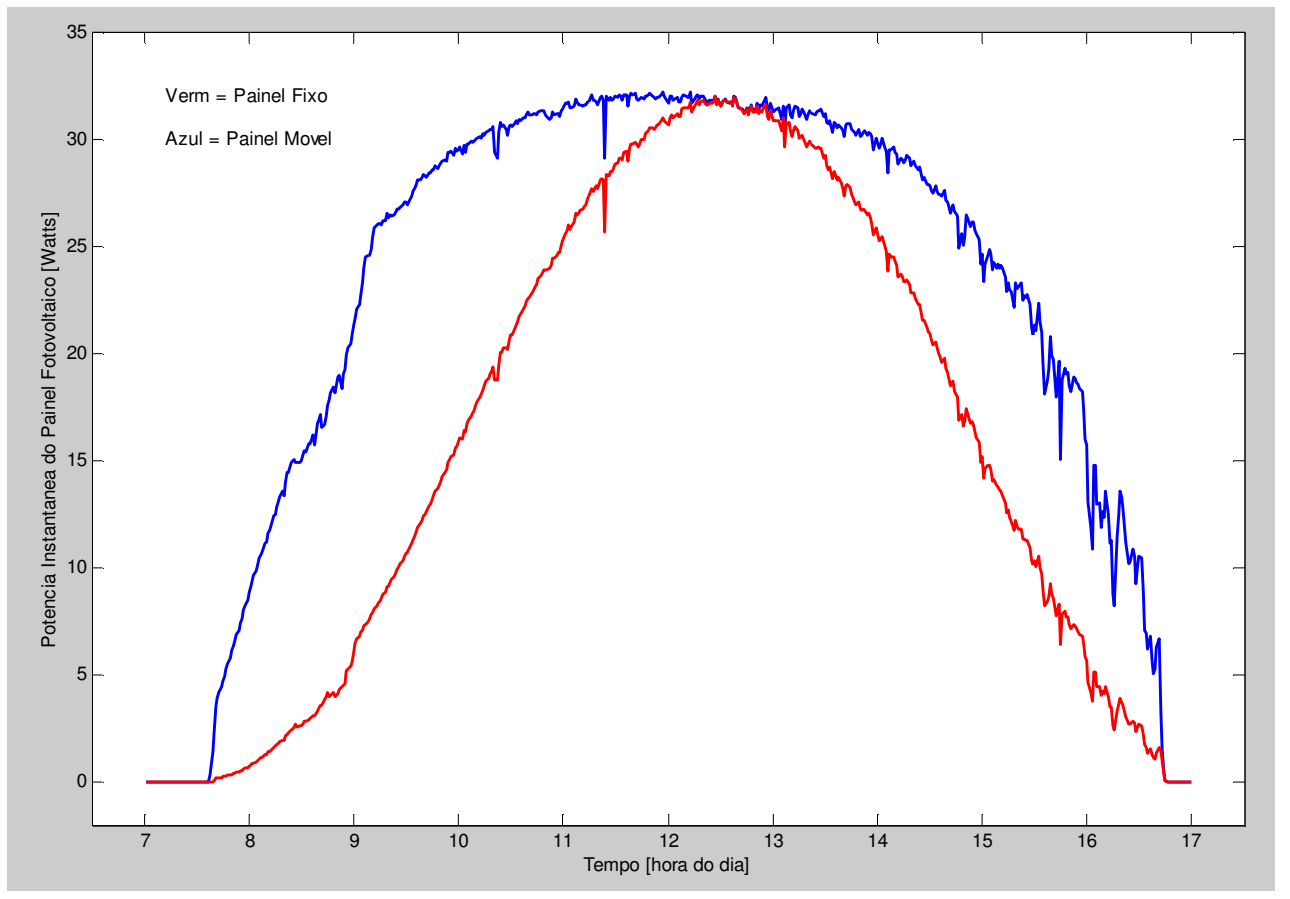

Figura 8 - Potência instantânea dos Painéis Fotovoltaicos - 26/06/2008.

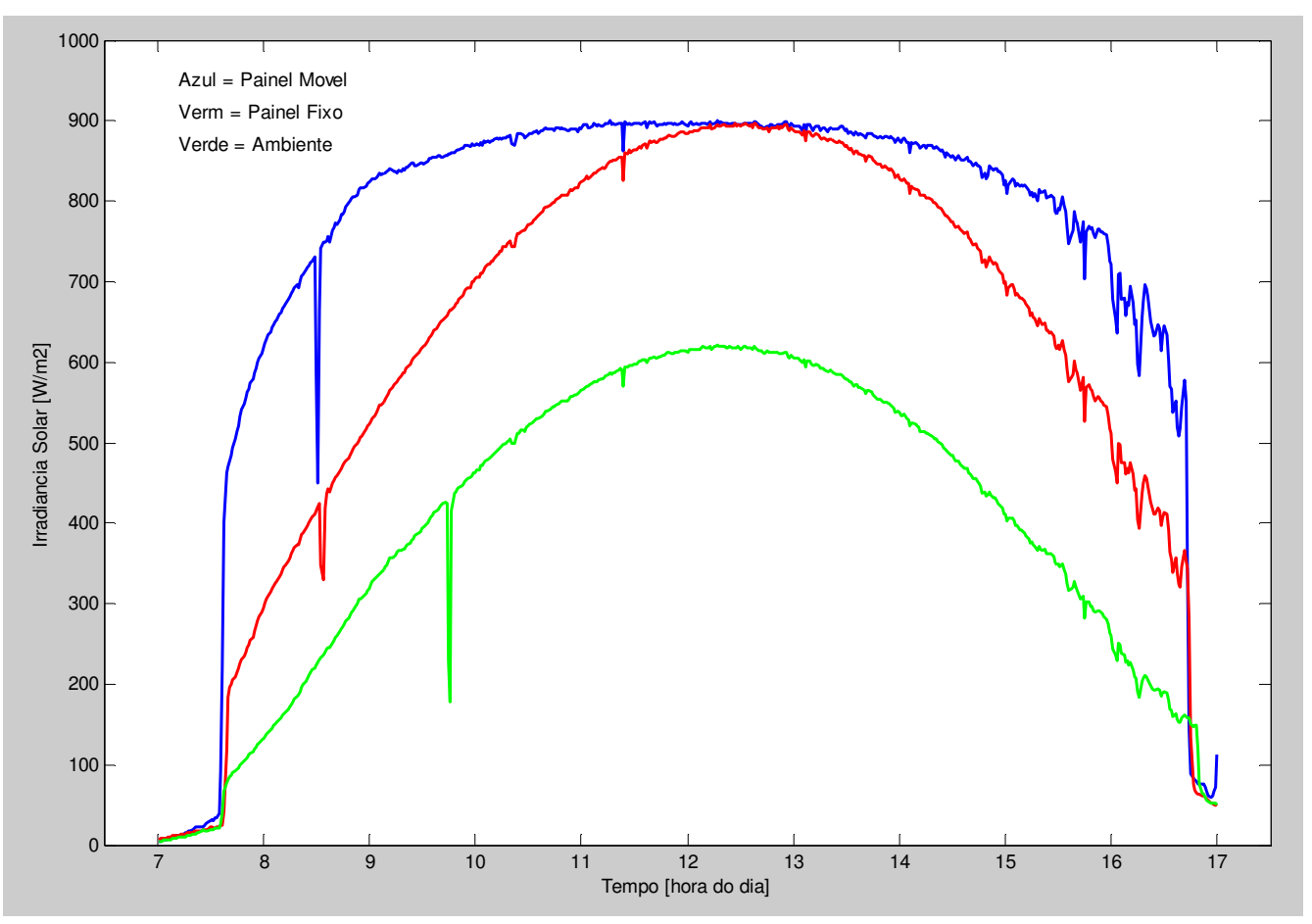

Figura 9 - Insolação incidente nos Painéis Fotovoltaicos - 26/06/2008. 
Integrando-se as curvas de potência ao longo do dia, o resultado da energia gerada é 156,5 Wh para o painel fixo, contra $225,0 \mathrm{Wh}$ para o painel móvel, o que representa um ganho bruto de $43,77 \%$ na geração de energia. A Figura 9 apresenta as curvas de insolação referentes ao dia 26/06/2008.

Um aspecto relevante é o valor máximo observado nestas curvas; por se tratar de inverno no hemisfério Sul, a Terra encontra-se mais distante do Sol, e o nível de potência solar que atinge a superfície é menor do que no verão. Nas curvas traçadas, o valor máximo é da ordem de $900 \mathrm{~W} . \mathrm{m}^{-2}$.

A Figura 10 apresenta as curvas de potência para os dois painéis operando em condições idênticas, com dados coletados entre $7 \mathrm{~h} 00$ e $17 \mathrm{~h} 00$ ( LCT ) do dia 03/03/2008.

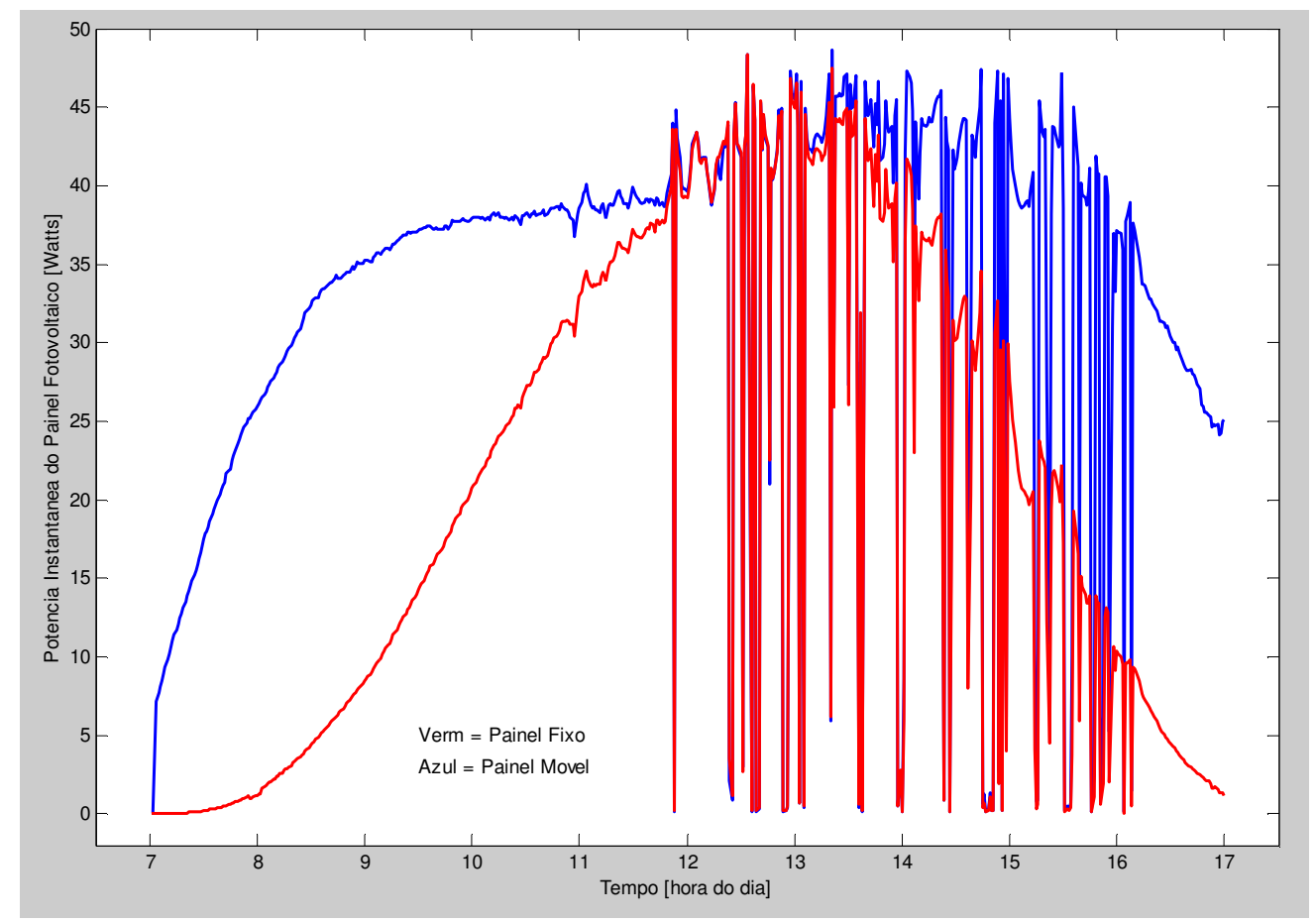

Figura 10 - Potência instantânea dos Painéis Fotovoltaicos - 03/03/2008.

Nestas curvas verifica-se o comportamento típico dos dias de verão na localidade onde estava instalado o Laboratório de Campo, onde se destaca o céu claro no período da manhã (o traçado da curva é 'suave', sem alterações bruscas nos valores de potência instantânea) e nebulosidade intensa no período da tarde (verificada pelos traços verticais indicando bruscas variações nos valores de potência, notadamente a partir das 12h00). Neste dia, não foram observadas chuvas, bastante comuns nesta época do ano.

Pelo fato do Sol nascer mais cedo, observa-se potência com valores significativos no painel móvel nos primeiros dados do dia, apresentando $17,4 \mathrm{~W}$ por volta da $7 \mathrm{~h} 30 \mathrm{~min}$. Ao final do dia, o sistema encer- 
rou sua coleta de dados às $17 \mathrm{~h}$, mas pelo último valor registrado ( $25 \mathrm{~W}$ para o painel móvel) conclui-se que ainda havia algum tempo de Sol.

Os máximos valores de potência foram 48,64 W (painel móvel) e 48,36 W (painel fixo), superiores aos valores registrados na Figura 8, que foram, respectivamente, 32,18 W e 31,97 W. Isto se deve à época do ano; sendo verão no hemisfério Sul, a Terra está mais próxima ao Sol, recebendo por este motivo mais energia em sua superfície. Conforme previsto, o painel móvel teve melhor desempenho em potência, chegando a atingir picos de 29,2 W acima da potência do painel fixo (295\% de ganho na geração de energia elétrica). Calculando-se a energia gerada, obtém-se 198,3 Wh para o painel fixo, contra 329,3 Wh para o painel móvel, o que representa um ganho bruto de 66,14\% na geração de energia. Observa-se ainda um aumento substancial da energia gerada em comparação com um dia de inverno, chegando-se a $+26,71 \%$ para o painel fixo e $+46,44 \%$ para o painel móvel.

As curvas de irradiância medidas no plano dos painéis e apresentadas na Figura 11, para o mesmo dia citado na Figura 10, apresentam como aspectos relevantes, além dos já citados, altos valores para as primeiras coletas de dados, registrando $627 \mathrm{~W} . \mathrm{m}^{-2}$ às $7 \mathrm{~h} 09 \mathrm{~min}$ e valores de pico bastante superiores aos observados em dias de inverno, chegando a atingir $1109 \mathrm{~W} \cdot \mathrm{m}^{-2}$ no painel fixo e $1125 \mathrm{~W} \cdot \mathrm{m}^{-2}$ no painel móvel, devido ao verão do hemisfério Sul.

A

Tabela 2 resume as médias apuradas durante o período de coleta de dados, entre fevereiro e julho de 2008. Estes resultados foram obtidos a partir da soma dos valores da energia gerada diariamente dentro de cada mês, dividida pelo número de dias de amostragem, o que resultou na Média Diária de Energia Gerada para cada um dos dois painéis. A comparação entre os valores médios gerados resultou na Média do Ganho na Geração, valor apresentado em porcentagem, por ser comparativo. Para completar este conjunto de dados, preencheu-se uma última linha referente às médias de cada coluna, permitindo o cálculo da Média do Ganho na Geração para o período analisado, resultando no valor de 53\%. 


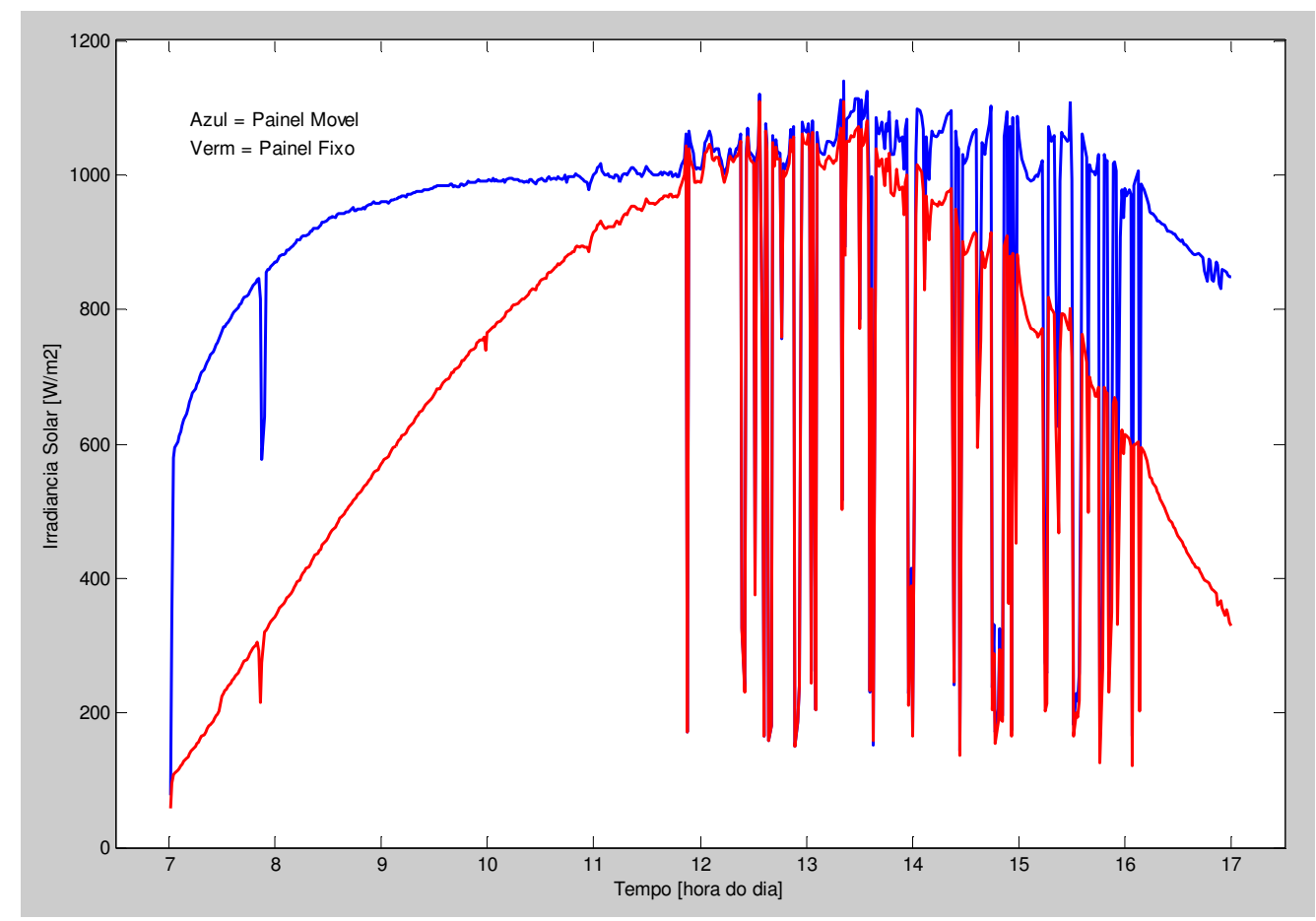

Figura 11 - Insolação incidente nos Painéis Fotovoltaicos - 03/03/2008.

Tabela 2 - Dados médios de geração de energia elétrica por ambos os painéis.

\begin{tabular}{cccc}
\hline Mês & $\begin{array}{c}\text { Média Diária } \\
\text { da Energia Gerada } \\
(\text { Painel Fixo - Wh) }\end{array}$ & $\begin{array}{c}\text { Média Diária } \\
\text { da Energia Gerada } \\
(\text { Painel Móvel }- \text { Wh })\end{array}$ & $\begin{array}{c}\text { Média do Ganho } \\
\text { na Geração } \\
\text { (Móvel/Fixo - \% })\end{array}$ \\
\hline Fev/2008 & 125,0197 & 196,0899 & 56,8471 \\
\hline Mar/2008 & 119,2039 & 197,2418 & 65,4659 \\
\hline Abr/2008 & 95,2888 & 149,0618 & 56,4317 \\
\hline Mai/2008 & 143,2470 & 217,2234 & 51,6426 \\
\hline Jun/2008 & 111,2681 & 156,3569 & 40,5226 \\
\hline Jul/2008 & 141,2527 & 208,6112 & 47,6866 \\
\hline Média & $\mathbf{1 2 2 , 6}$ & $\mathbf{1 8 7 , 4}$ & $\mathbf{5 3 , 1}$ \\
\hline
\end{tabular}


No experimento montado, a alimentação do CLP e do sistema responsável pelo movimento foi obtida a partir da rede convencional de energia, quando em um sistema real, não-experimental, esta energia deverá ser suprida pelo próprio aparato fotovoltaico juntamente com seu banco de baterias.

Com a finalidade de se avaliar o ganho líquido na geração de energia, mediu-se o consumo dos motores, dos circuitos eletrônicos e do conjunto CLP + expansões. Como resultado, encontrou-se um consumo de 89,38 Wh para o sistema de movimento, o que representa $65 \%$ da energia gerada, no pior caso, tornando a proposta inviável. Entretanto, há de se considerar que o sistema de movimentação não necessita do CLP, pois não há a necessidade de coleta de dados, mas apenas de controle do movimento. O programa de movimentação pode ser implantado em um microcontrolador dedicado, por exemplo, da família PIC, de baixo consumo e compatível com a aplicação proposta. Tomando-se como exemplo o microcontrolador PIC 16F877, a partir de seus dados de catálogo, encontra-se um consumo máximo de energia correspondente a apenas $0,11 \%$ da energia gerada no pior caso. Considerando-se ainda a necessidade de memória adicional para o controlador e o consumo de seus periféricos, sempre da ordem de mili-Watthora, o consumo total nunca ultrapassará os $0,2 \%$ da energia gerada.

Sob o ponto de vista do melhor aproveitamento da energia solar, os resultados apresentados demonstram a superioridade do sistema móvel em relação ao fixo. Entretanto, a implementação proposta apresenta-se mais complexa. Analisando-se as despesas com materiais, construção e implantação de ambos os sistemas, verificou-se um custo 35,7\% superior para o sistema fotovoltaico móvel. Entretanto, este custo maior é compensado pelo ganho médio na conversão de energia, da ordem de 53\%.

Nesta situação, calculando-se a relação entre o custo de cada um dos sistemas pela sua geração média diária de energia, conclui-se que o retorno do investimento realizado para implantação de um sistema fotovoltaico com posicionamento automático ocorre em um tempo 11,23\% inferior ao tempo necessário para recuperação do investimento realizado na instalação de um sistema fotovoltaico fixo.

\section{CONCLUSÕES}

A pesquisa realizada atingiu os objetivos propostos, tendo sido desenvolvido um sistema de posicionamento automático para painéis fotovoltaicos e avaliado o seu desempenho quando comparado ao desempenho de um sistema fotovoltaico fixo, ambos operando sob as mesmas condições de insolação e carga elétrica.

Neste trabalho foram verificados resultados do aumento na energia gerada de até $70 \%$ para determinadas situações, com um valor médio de 53\%, sendo que o consumo dos componentes eletromecânicos responsáveis pelo controle e movimento do painel representa menos de $0,1 \%$ desta energia. 
Uma importante contribuição que a pesquisa apresenta refere-se ao suporte mecânico desenvolvido, o qual posiciona o painel com dois graus de liberdade, entretanto movimentando apenas um motor ao longo do dia; isto resulta em significativa redução na complexidade do programa de controle, além de proporcionar menor consumo de energia para a movimentação do painel.

A não utilização de sensores para determinação da posição aparente do Sol representa inovação tecnológica, pois evita movimentos desnecessários em casos de sombras causadas por nuvens, por exemplo. O programa de controle prevê com exatidão a localização do Sol no céu e aciona os motores de passo de modo a direcionar o painel solar em posição perpendicular aos raios incidentes.

O uso do CLP para controle e monitoramento mostrou-se adequado para a atividade de pesquisa; entretanto, devido a seu custo, dimensões físicas, e principalmente, consumo de energia, demonstrou não ser a melhor solução para um sistema de movimento como este. A construção de um controlador dedicado atinge melhor os objetivos de simplicidade e baixo custo, apresentando baixo consumo de energia.

A análise dos custos de implantação permitiu concluir que o retorno do investimento em um sistema móvel ocorreu em tempo inferior ao tempo necessário para recuperação do investimento realizado na instalação de um sistema fotovoltaico fixo, justificando sua viabilidade econômica.

Dentro deste contexto, concluiu-se que o sistema proposto é uma alternativa viável, tanto do ponto de vista econômico quanto das vantagens na conversão de energia e simplicidade de implantação, para o fornecimento de energia elétrica para domicílios rurais de baixo consumo, ou consumidores em áreas isoladas, para os quais a extensão da rede elétrica convencional signifique alto custo ou apresente dificuldades de ordem técnica. Além disso, a pesquisa desenvolvida representou uma contribuição aos estudos na área de sistemas fotovoltaicos isolados de pequeno porte, por apresentar soluções simplificadas como a não utilização de sensores para o posicionamento, o uso de motores de passo e um novo modelo de suporte mecânico para 2 graus de liberdade, com movimento alternado dos motores.

\section{AGRADECIMENTOS}

Os autores agradecem à Fundação de Amparo à Pesquisa do Estado de São Paulo - FAPESP e à Fundação para o Desenvolvimento da UNESP - FUNDUNESP, pelo apoio financeiro, e à direção da FE/UNESP-Bauru, pela cessão do espaço para instalação dos equipamentos. 


\section{REFERÊNCIAS}

ATLAS de energia elétrica do Brasil. 2. ed. Brasília, DF: Agência Nacional de Energia Elétrica, 2005. $243 \mathrm{p}$.

BRASIL. Ministério de Minas e Energia. Empresa de Pesquisa Energética. Plano nacional de energia 2030. Brasília, DF, 2007. Disponível em: <http://www.epe.gov.br/PNE/20080512_9.pdf>. Acesso em: 18 set. 2008.

CHOJNACKI, J.; TENETA, J. Control algorithms for a remote sun-tracking photovoltaic system. In: EUROPEAN PHOTOVOLTAIC SOLAR ENERGY CONFERENCE, 19., 2004, Paris. Proceedings... Paris, França: WIP-Renewable Energies, 2004. p. 2219-2222.

CHUN-SHENG, W. et al. Study on automatic sun-tracking technology in PV generation. In: INTERNATIONAL CONFERENCE ON ELECTRIC UTILITY DEREGULATION AND RESTRUCTURING AND POWER TECHNOLOGIES, 3., 2008, Nanjing. Proceedings... Nanjing, China: IEEE, 2008. p. 2586-2591.

ITO, M. et al. An analysis of variation of very large-scale PV (VLS-PV) systems in the world deserts. In: WORLD CONFERENCE ON PHOTOVOLTAIC ENERGY CONVERSION, 3., 2003, Osaka. Proceedings... Osaka, Japão: IEEE, 2003. v. 3, p. 2809-2814.

ITO, M. et al. Comparative study of fixed and tracking system of very large-scale PV (VLS-PV) systems in the world deserts. In: EUROPEAN PHOTOVOLTAIC SOLAR ENERGY CONFERENCE, 19., 2004, Paris. Proceedings... Paris, França: WIP-Renewable Energies, 2004. p. 2113-2116.

MARINESCU, D.; MARINESCU, C. Control optimizing algorithm for soft sun-trackers. In: IEEE INTERNATIONAL CONFERENCE ON AUTOMATION, QUALITY AND TESTING, ROBOTICS, 2006, Cluj-Napoca. Proceedings... Cluj-Napoca, Romênia: IEEE, 2006. v. 1, p. 54-57.

STINE, W. B.; GEYER, M. Power from the Sun. 2001. Disponível em: <http://www.powerfromthesun.net>. Acesso em: $18 \mathrm{dez} .2006$. 